\title{
Using time-resolved crystallography and cryo-EM to investigate human DNA repair nucleases
}

Lorena S. Beese ${ }^{1}$, Yuqian Shi, and You Wang

Department of Biochemistry, Duke University Medical Center, Durham NC 27710.

1 lorena.beese@duke.edu

Human Exonuclease I (hExo1) plays key roles in DNA repair, replication, and recombination. Defects in hExo1 are linked to various cancers. It exposes large singlestranded regions by processively excising nucleotides from 5' ends of nicks or breakage points; it also removes 5' flaps by endonucleolytic cleavage. Control of these activities is critical for proper hExo1 function. Time-resolved crystallographic studies have shown that processive cleavage involves a carefully orchestrated sequence of protein conformational changes and DNA motions that iteratively place scissile bonds into the active site for hydrolysis catalyzed by two $\mathrm{Mg}^{2+}$ ions, release the excised nucleotide or flap, and reset the protein for the next reaction cycle by translocating the enzyme along the DNA $(1,2)$. Here we expand our time-resolved X-ray crystallography studies to define reaction intermediates and describe a novel, inhibited state observed in several exo- and endonucleolytically cleaved (processed) and uncleaved (initiation) DNA complexes. Structures of hExol complexes with mismatch repair proteins Human Msh2Msh6 and MSH2-Msh3 heterodimers define critical interaction interfaces essential for repair.

\section{References}

(1) Orans, J., McSweeney. E.A., lyer, R.R., Hast, M.A., Hellinga, H.W., Modrich, P, \& Beese, L.S. (2011) Structures of human exonuclease 1 DNA complexes suggest a unified mechanism for nuclease family. Cell April 15;145(2):212-23. (PMCID: PMC3093132).

(2) Shi Y, Hellinga HW, Beese LS. (2017) Interplay of catalysis, fidelity, threading, and processivity in the exo- and endonucleolytic reactions of human exonuclease. Proc Natl Acad Sci U S A. 2017 May 22; 114(23):6010-6015 (PMID: 28533382). 